\title{
Security Aspects on Inter-Organizational Cooperation Using Wrapper Agents
}

\author{
Bengt Carlsson, Paul Davidsson, Andreas Jacobsson, \\ Stefan J Johansson, and Jan A Persson \\ Blekinge Institute of Technology, School of Engineering \\ 37225 Ronneby, Sweden \\ $\{$ bca, pdv, aja, sja, jps\}@bth. se
}

\begin{abstract}
The significance of electronic information exchange in interorganizational cooperation is well-known. We will here focus on the particular requirements of SMEs. We describe a general "wrapper agent" solution based on open source freeware that makes it possible (in principle) for any business system to exchange information with any other business system. It has been successfully applied in a pilot study involving two companies in a transport chain using different business systems. We also suggest further improvements by addressing security and privacy issues as well as an extended, possibly dynamic, set of involved companies and higher levels of cooperation.
\end{abstract}

\section{Introduction}

Most organizations recognize the critical role that IT plays in supporting their business objectives as well as fueling profitability and growth. As more and more small and medium sized enterprises (SME) adjust their businesses to the increasing demands for IT solutions, there is a set of criteria that should be met. Some of these are; interoperability (both within the enterprise and with other enterprises), scalability, adaptability (handling shifting customer needs), independence (avoiding technological lock-in or path-dependency), cost-effectiveness, simplicity and usability, as well as security and privacy (preventing unintended exposure of sensitive information). We will here focus on inter-organizational cooperation and we discriminate between three dimensions of such cooperation:

1. The level of cooperation: This is related to the content and purpose of the exchanged information with tasks ranging from administrative information exchange to complex operation planning. An example of a simple task is ordering and invoicing, whereas a more complex task may concern making critical information available to the cooperating partners, in order to improve operations by more efficient planning and scheduling.

2. The number of involved enterprises: The more parties involved in the cooperation, the more complex solutions are needed. The simplest case concerns cooperation between only two enterprises (one-to-one cooperation), whereas the 
general case involves a large number of enterprises cooperating with each other in different ways (many-to-many cooperation).

3. The dynamics of the cooperation community: In the simple static case, the parties involved are known from the start and will not change. In a truly dynamic cooperation, on the other hand, participants may join and leave the cooperation at any time with little time left to build trustful relationships.

Recent work by Davidsson et al. describes a decentralized solution that meets most of the requirements listed above by using wrapper agents [4]. In principle it can be used for all types of inter-organizational cooperation, but has currently been applied only to the simplest case (according to the three dimensions identified above), i.e., information exchange between two enterprises in a static environment. The wrapper agent solution increases efficiency and productivity by enabling simplified administrative tasks, and by improving business operations. It was originally tested in a transport chain setting, however, we believe that the wrapper agent approach is of a general nature. Here, we will analyze the security and privacy aspects of this approach.

The next section describes central concepts and related work and in Section 3 we present the wrapper agent solution that was developed to support and facilitate information exchange. Section 4 describes one simple and one complex case of cooperation, which in section 5 are discussed from a security and privacy point of view. Finally, some conclusions are drawn and suggestions are made for future work.

\section{Concepts and Related Work}

The review includes existing software solutions available on the market, a conceptual overview of the security, privacy and authentication aspects addressed within the wrapper agent solution, and a short characterization of transport chains.

\subsection{Existing Technology}

Support for electronic information exchange is often found in large business systems, e.g., ERP (Enterprise Resource Planning) systems. Such systems often focus on a specific type of company, e.g., a producer in the process industry or a transport operator. The systems do often have some ability to exchange information with other types of business systems, but this is often limited to a standard set of formats and systems. There exist several off-the-shelf TA (Transport Administrative) systems for storage, synthesis and communication of data, such as Movex [9], Hogia MobiLast [7] and many more. These systems and the other systems that support electronic data interchange have varying characteristics, but all of them require substantial investments by the user. As a consequence, SMEs are able only to invest in one system, if any at all. This, in turn, makes it impossible to carry out electronic business activities with several companies using different business systems due to system interoperability problems.

One existing solution that meets some of the interoperability requirements is Microsoft BizTalk [2]. The main purpose with BizTalk is to facilitate system 
communication independently of the individual communication formats in the systems by acting as an interpreter between the systems. It is based upon a central server through which all exchanged information pass. It uses XML and supports the main protocols for email and http. However, being a proprietary client-server solution it has several disadvantages, such as, making the actors dependent of third party, being expensive and having possible risks for communication bottlenecks.

\subsection{Security and Privacy}

Today's highly connected IT infrastructures exist in an environment which is increasingly hostile - attacks are being mounted with growing frequency and are demanding ever shorter reaction times. Due to an increase in the amount of information that is being made openly available, the risks of unsolicited, unintended or malicious use have augmented. Often, organizations are unable to react to new security threats before their businesses are impacted, let alone recover from any damage. In a setting where more and more aspects of organizations are being adjusted for IT, it is seemingly problematic to keep the overall IT related risks in control. Managing security of IT infrastructures, and the business value that those infrastructures deliver, has become a primary concern for most IT departments. Violations on IT infrastructures occur in numerous ways throughout the Internet and both small and large organizations need to consider internal (e.g. insiders) and external threats (e.g. hackers, rival competitors, etc.). A large supply of privacyinvasive and malicious software is already available. Malware, that is malicious code planted on computers, gives attackers a truly alarming degree of control over systems, networks and data. For instance, when spyware programs are involved, the loss in control of sensitive corporate information, pose a threat to distributed (or shared) information resources. The unauthorized user gets confidential information either directly or indirectly by utilizing an infiltrated system for additional attacks. Letting go of control increases the risk for security violations.

Information security is about preventing adverse consequences from the intentional and unwarranted actions of others [10]. Typically, information security services ensure confidentiality, integrity and availability ${ }^{1}$. Although information security is by no means strictly a solution to a technical problem, its technical aspects (firewalls, authentication mechanisms, encryption techniques, etc.) are important. Managing information security is an increasingly high-profile problem, as hackers, malicious actors and rival competitors may take advantage of the fact that organizations are opening up parts of their systems to employees, customers and other businesses via the Internet. This is very much the case with SMEs when they go digital.

Privacy $^{2}$ is normally not limited only as a right for individuals, but also for institutions (e.g. a company) and/or groups of individuals. The dilemma of finding a balance between risks in terms of the extent of available information and opportunities in terms of business utility is a well known problem.

\footnotetext{
${ }^{1}$ However, other services like, e.g. non-repudiation, could also be included.

${ }^{2}$ We use the concept of privacy in conformity with the definition by Alan F. Westin: "Privacy is the claim of individuals, groups and institutions to determine for themselves when, how and to what extent information about them is communicated to others" [12].
} 
Within the wrapper agent solution presented Section 3, two technical measures are used in order to enable privacy and security, namely authentication and encryption. Authentication is used to confidently associate an identity with a wrapper agent, person or entity in an organization [11]. A well-designed authentication system allows agents, users or organizations to prove their identities conveniently and to gain access to a network without threatening the safety of the organization. Encryption is the process of scrambling a message so that it can only be read by the party it is intended for [1]. Typically, business transactions on the web and email messages are encrypted, for security or privacy reasons. This is also the case for the messages sent or received by the wrapper agents. Authentication in combination with encryption mechanisms addresses privacy, and three aspects of information security: confidentiality, integrity and non-repudiation [11].

Interoperability solutions convey numerous security and/or privacy threats, which are difficult to neutralize using only authentication and encryption. Among many things, SMEs must also hold other security mechanisms as well. The SME holding the poorest security settings, i.e. lacking firewalls, anti-virus software, having weak authentication mechanisms, etc., constitutes a great threat to other parties in the cooperation alliance. In general, the consequences of being exposed to intrusions, attacks, deception and infiltration may be loss and/or tampering of data and/or system resources, as well as unnecessary costs for network and system maintenance. Another aspect concerns data access. For instance, a result of users that interact with other business systems of the cooperating parties may be that they gain a non-intentional all-or-nothing access to other available databases, which may contain sensitive data.

A discussion concerning security and privacy aspects of the interoperable wrapper agent solution can be found in Section 5.

\subsection{Characteristics of Inter-Organizational Transport Chains}

A transport chain usually involves a large number of actors, and in order to reach an acceptable level of efficiency it is important to synchronize these actors and their activities. For example, information concerning the status of the cargo needs to be available to the right person, at the right place, and at the right time. Although this is obvious for everyone involved and that advanced technology to collect and distribute information exists, this is seldom achieved. We argue that the main reasons are not due to lack of solutions, but rather due to difficulties in integrating existing software systems and the associated high costs. Instead, a large amount of transport-related information, such as bookings and cargo specification, is exchanged manually via fax machines and phones. Problems that arise due to the manual work required are for instance that information may not be accessible at a certain place when needed and also information duplication is complicated. The question that remains to be answered then is: are advanced and expensive systems required to solve these problems, or are there other, simpler and less expensive, solutions?

In the project "Transport informatics for increased cooperation between the parts in a logistic chain", the overall aim was to develop and demonstrate the use of a common platform where the companies are able to exchange the necessary 
information between themselves as well as their other customers and suppliers [4]. The platform and its functionalities was later demonstrated on one of the transport chain case studies and evaluated. Besides the software platform, the project also generated insights in the problems related to technological aspects as well as organizational issues within the freight transportation industry.

\section{The Wrapper Agent Solution}

As mentioned in the introduction, some of the most important needs for improvements were simplifications of administrative activities, such as, transport booking, tracing of goods and carriers, deviation detection, and calculations of environmental load. Many of these require a complete unbroken process of gathering data, data processing and information distribution. In a pilot study we decided to focus on the reduction of usage of fax machines and other manual ways of information exchange (reducing the administrative costs as well as the number of errors caused by the human factor) and increase the accessibility of information by making electronic information exchange possible [4].

A software prototype was built using only freeware with open source code such as, Java, MySQL, J2EE, JBoss, etc., and state-of-the-art technologies like XML and web services. The prototype provides the possibility for different business systems to communicate and support information exchange via web portals, email, fax and SMS. The prototype is an information carrying system meaning that the system acts independently of what type of data that is transferred through it.

The basic idea can be seen as a generalization of the well-known Adapter design pattern [6] used within object-oriented software engineering, corresponding to the concept of wrapper agents [8]. It is also similar to the concept of connectors [3]. To each (legacy) business system a wrapper agent is built that enables the system to interact with the other business systems in the transport chain. Such a wrapper agent is mainly composed by three parts; a bridge, an interpreter, and a message handler. (see Fig. 1) The bridge handles the interaction with the business system, and the interpreter translates the data from the format that the sending business system is using to the format which the receiving business system is using (and vice versa). The message handler takes care of the communication with the adapters of the other business systems. The bridge typically makes use of the functions for exporting and importing information that most business systems are equipped with. If this is not the case, more sophisticated methods must be used.

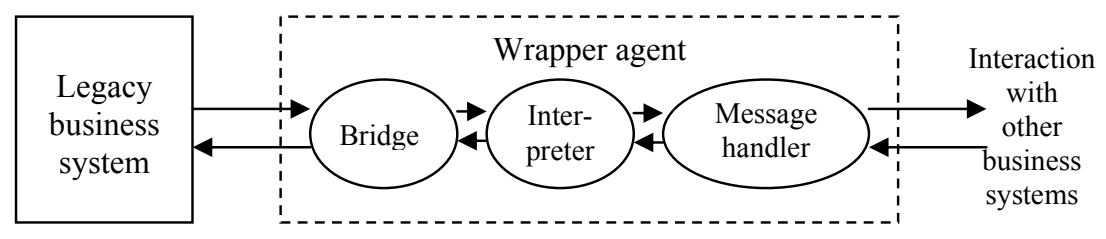

Fig. 1. The structure of the wrapper software. 
The wrapper prototype software is available as freeware and is relatively easy to install. It includes general message handling components and shells for the interpreter and the bridge. Much effort was spent on reusability making it easy to adapt the software to arbitrary business systems (at least those that have basic data import and export functionalities). Also modifiability was considered and since the software is module-based, extensions of the system are facilitated. The system requires an authentication mechanism ("log in") for usage, and encryption of data as well as digital labeling increase the degree of security and privacy.

\section{Case Studies}

Below we present two case studies. The simpler case (case A) involves information exchange in a static environment containing two enterprises whereas a more complex case (case B) uses an, at least partly dynamic, environment with several enterprises, making activities such as planning and scheduling more efficient.

\subsection{The Study of Case A}

The transport chain that was selected for the case study was not very complicated. It consisted of just two main actors and little or no cross-border traffic. The focus was on SMEs based in the region of Blekinge, Sweden, with partly very limited resources for investments in IT but they still needed the functionalities to stay competitive. Many of the needs for improvements and opportunities identified in the specific case studies were also found in other companies in the transportation sector with a variety in size, nationality and resources. An extended description of the participating actors and their identified motives is found in [4].

The prototype platform was tested by having two different major business systems; Hogia Mobilast and Movex, in two different companies, Karlshamns Expressbyrå (KE) and Karlshamns $\mathrm{AB}(\mathrm{KAB})$, interacting via the platform (see Fig. 2). In the demonstration experiments the actual business systems (together with the associated adapters) were executing at the two companies. A number of functionalities were tested. For instance, KAB was able to make transport requests from Movex directly into the Hogia Mobilast system at KE. Similarly, KE was able to confirm the request from inside its system directly to Movex at KAB. Further functionalities include monitoring active requests. 


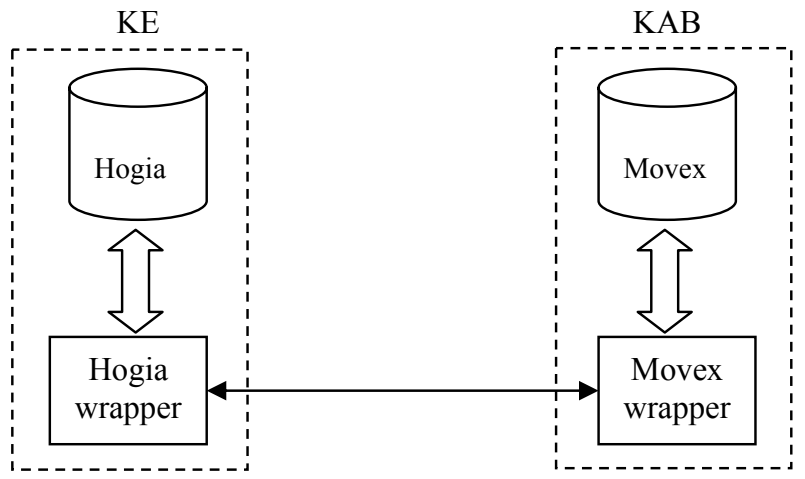

Fig. 2. The setting of case A.

The most complex part of a wrapper agent is typically the Bridge. As an example, we take a closer look at the Bridge of the Hogia Mobilast wrapper agent which is composed of three threads:

- Import, which receives XML messages from the Message handler via a web service client, and saves them as files in the "In" folder.

- Export, checks the "Out" folder every fifth minute (this time interval was used in our experiments; it should, of course, be chosen based on the response time requirements for the particular application) and when a new file is detected, it is sent via a web service client to the Interpreter for further processing. When it gets an acknowledgement from the Message handler that the message was successfully sent, the file is moved from the Out folder to a "Sent Orders" folder.

- Status monitor, monitors changes regarding the orders that are currently active, by making requests concerning the status of these orders each 20 second (in our experiment) and saves them as XML-requests to the In folder of Hogia Mobilast. It then reads the reply from the Out folder and sends it to Interpreter for further processing.

Bridges for other business systems with import and export facilities work in a similar way.

\subsection{The Study of Case B}

In the research project "Integrated production and transportation planning within the food industry", a transport chain consisting of one producer, one transport operator, and a number of customers is studied [5]. The project aims at achieving improved performance (cost reduction) through the development and usage of advanced planning tools. A system perspective is taken which requires a higher level of 
cooperation and information exchange between the actors than in the first case study. The studied chain consists of the producer KAB, the transport operator FoodTankers, and typical customers to $\mathrm{KAB}$. In the studied transport chain, it is customer orders that drive the transportation and production planning. Currently, the first planning step is to decide whether a new order can be accepted or not. The subsequent production planning is about deciding when and in which order the products should be produced; and about planning the inventory storage. The transport planning concerns meeting the transport requirements specified by customer orders obtained via the producer. When transport orders appear which require high cost solutions (or the planner can deliver significant cost savings by replanning), the transport planner may suggest changes to the producer which in turn check for such changes with the customer. This implies that there are tentative plans and cost implications shared between the partners.

Currently it is hard to alter the delivery times and quantities of transport orders. One reason for this is the lack of information about the "true demand" of the customer, e.g. safety stock levels and consequence of delivering earlier or later than requested. Another reason is the lack of decision support tools for capitalizing on this potential additional information. In the studied transport chain it has been observed that the level of information sharing/exchange is different between different customers and the producer and between different customers and transport operator. Moreover, now and then some new customers join the transport chain and others leave it. It is believed that in order to significantly improve the performance at least some of the customers need to exchange more information with the producer and the transport operator.

\section{Discussion}

We explore the effects on security and privacy caused by the wrapper agent solution to inter-organizational cooperation. The fact that the system is computerized implies that there is at least just an as large threat to information security for SMEs that adapt to the system as there are for SMEs using alternatives such as web services, email, etc. However, while the wrapper agent system has some clear advantages in that it supports scalability, adaptability, undependability, cost-effectiveness, usability and interoperability, we must also carefully consider what specific risks that are conveyed by this particular system. Privacy and security risks within the wrapper agent system can be managed (though not necessarily eliminated) by utilizing authentication and encryption mechanisms since they are designed to protect privacy and security (confidentiality, integrity and non-repudiation).

\subsection{Security and Privacy Aspects}

Based on the two case studies (one simple, case A, and one complex, case B), we will now discuss how security and privacy are affected. In particular, we will investigate this based on the three dimensions of cooperation mentioned in the introduction. 


\subsubsection{The Level of Cooperation}

Pure information exchange processes are normally not a privacy violation problem, but a security problem. Here, security concerns are focused on ensuring data integrity, confidentiality and non-repudiation. In the wrapper agent solution this is solved by implementing authentication and encryption, as mentioned in 2.2. In addition, general security configurations should be included within any SME involved in a cooperation alliance. The weakest link in a scenario involving wrapper agents is the SME that holds the poorest security settings, i.e. ignoring firewalls, anti-virus software, and having weak authentication mechanisms among many things.

In the wrapper agent solution, partners might gain unintentional access to other available databases, which may contain sensitive data. The unintentional revealing of information is accentuated when shifting from solely information exchange functions into information sharing for operations planning with another party since more information is shared. Further, since cost implications on tentative plans might be shared, other partners may build up information profiles about its partners which is critical for the business (e.g. a transport operator's exact costs of operations might be revealed). In terms of privacy, it may be difficult to control what information is obtained by the other parties, as well as how and to what extent it is used. This may create an unbalance in the business relationship between the SME and other companies, since the information can be used in for example business negotiations about terms of contracts.

\subsubsection{The Number of Involved Enterprises}

In a second scenario, where there is a shift from a one-to-one to a many-to-many relationship between the interacting parties, there is an exponential growth of privacy related conflict of interest. Problems arise in that the difficulties for guaranteeing that information, which may be sensitive to one party, is not being misused by rival competitors or malicious actors. Also, when many companies are involved in the wrapper agent system, access of available data is more difficult to restrict than if there is a one-to-one relationship. The risks for unintentional or intentional violation to privacy increases as the network of participating entities grow.

Here, security mechanisms must protect against attacks, deception, intrusions, and insiders so that confidentiality and integrity can be ensured. If security solutions are proven efficient over time, trust between the different parties is enabled, which is necessary for the prosperity of a wrapper agent network.

\subsubsection{The Dynamics of Cooperation Community}

An extension of the many-to-many scenario is when the case changes from static to dynamic, i.e. participants may join or leave the setting. This shift creates similar but not identical security and privacy problems as in the scenarios above, although the consequences here are a bit more problematic to analyze.

Complexity in terms of security and privacy is increasing due to that the number of participating parties changes dynamically. The level of cooperation between the involved entities may be fluctuating, resulting in problems with restricting data access over time in-between a dynamic environment of companies. This implies that the participants may have access to information exchanged, even though their cooperation 
with the wrapper agent system is terminated, due to inconsistencies in the system. This may affect the other parties within the system in that sensitive business information may be distributed or used by unauthorized companies for their own purposes. Hence, dynamic cooperation increases the difficulties in ensuring privacy.

Besides keeping track of authorized users and of the data in use, the wrapper agent system grows and diminishes as the number of entities changes, which among many things makes it hard to build trustful relationships. At the same time, threats of deception, infiltration and attacks get accentuated, rendering in that general security configurations of involved parties are crucial for enabling trust. Malicious actors may otherwise gain access to or attack the wrapper agent system by launching, e.g. malware via an SME holding poor security configurations. In particular, confidentiality and integrity are difficult to enhance when cooperation parties changes dynamically.

However, one respect that is not covered in the wrapper agent system is the time limit for data. If we could terminate data accessed within the system when an SME ends it cooperation with the system, the long-term and negative consequences to privacy and confidentiality could be mitigated. Perhaps, this would also lead to a better platform for enabling trust, since SMEs do not risk letting go in control of their most precious information resources.

\subsection{Direct use, Wrapper Agents and Centralized Systems}

The suggested wrapper agent solution has some different characteristics compared to a centralized server solution such as BizTalk [2]. With respect to the security problems outlined above, there appear to be no imperative problems with the wrapper agent solution (compared to a centralized server solution). If no wrapper agent or centralized server solution is used, the control of the information to be exchanged is typically handled by the actors directly and locally when for instance sending faxes, phone calls, and email messages. A wrapper agent solution might change this situation less with respect to the level of control experienced by the actor than a centralized server solution controlled by another actor (or controlled by a third-party). Some security and privacy aspects might be easier to ensure using the wrapper agent solution than using a centralized server since such a solution is probably closer to the ways many SMEs currently manage their security and privacy (e.g. individually for each actor it is exchanging information with). Another great benefit is that there is no risk of a single point of failure with a decentralized system, as there is with a centralized one.

We have demonstrated the possibility to achieve basic and sufficient communication functionalities like the large-scale TA systems offer, but to a considerably lower cost with no apparent/obvious loss in security or privacy. This is something that is of significant importance to the survival of smaller actors with same needs as the larger players but with a much more limited investment possibility. 


\section{Conclusions}

An evaluation of the practical relevance and economical gain of the prototype was made based on interviews of the participating companies. The prototype provides a direct use since it offers cost-effective (due to the use of freeware and easiness of adaptation) and platform-independent communication. Thus, the prototype seems to perform well with respect to attributes such as interoperability and undependability with several interfaces. These benefits make the prototype useful in numerous contexts and not only for the project participants. A cost-effective communication solution generates in addition several implicit benefits such as a possible reduction of error in documents that are exchanged (due to minimization of manual duplication work) and increased information accessibility which have costs in terms of loss in control of sensitive data.

The wrapper agent solution includes basic security and privacy settings by means of authentication of the user and encryption of transmitted data for case A. Case B uses operations planning where partners join or leave in a many to many coalition. We discuss both internal and external security and privacy issues when handling such a complex environment.

Compared to existing centralized solutions, such as BizTalk, the proposed solution has a number of advantages, e.g., being independent of third parties and avoiding central communication bottlenecks.

\section{Future Work}

In order to solve the discussed security and privacy deficiencies, we should look at both organizational and technical solutions. Organizational settings are most probably valid within a wide range of organizations and that is beside the scope of this article. Technical solutions are either part of SME's general security configurations or part of the wrapper agent implementation. We will focus on the latter issue.

The wrapper agent solution, as described in Section 5, focuses on the tasks directly connected to achieve interoperability, but nothing restricts it from having additional tasks to fulfill. For instance, by extending the number of threads in the bridge (Fig. 1), we may include security checking of the XML messages sent to the bridge. Similarly, the message handler may place restrictions on the communication with the wrapper agents of the other business systems.

Given the three dimensions of inter-organizational cooperation, parts of the security problems may be solved by an extended wrapper agent solution. Unauthorized use of data by entrusted users is restricted by the message handler. A single SME has access to a restricted set of information. If an intruder uses an entrusted user for attacking the legacy business system, the bridge keeps a check on validation data for the file. How and to what extent this is done, has to be further explored. The important thing is the ability to include security checking. Finally, unauthorized access to the system is a more general question not addressed by a restricted SME community. 
We are currently working with applying the prototype to additional companies and business systems within the project "Integration between different SMEs business systems" financially supported by "Sparbanken Kronan", a national Swedish bank foundation. Moreover, we have been investigating the possibilities of developing an (or using an existing, such as the one suggested by [13]) ontology so that a common communication language can be used between the wrapper agents. This would significantly reduce the need of developing interpreters, since the communication of each business system only needs to be translated into one common language, independently of which business system it will interact with.

\section{References}

1. R. Anderson, Security Engineering - A Guide to Building Dependable Distributed Systems, John Wiley \& Sons, New York NY, 2001.

2. BizTalk home page, http://www.microsoft.com/biztalk/

3. Bures, T., Plasil, F., Communication Style Driven Connector Configurations, Software

4. P. Davidsson, L. Ramstedt, J. Törnquist, Inter-Organization Interoperability in Transport Chains Using Adapters Based on Open Source Freeware, Interoperability of Enterprise Software and Applications, in press, Springer Verlag, 2005.

5. Integrated production and transportation planning within the food industry home page, http://www.ipd.bth.se/fatplan/

6. E. Gamma, R. Helm, R. Johnsson, and J. Vlissides, Design Patterns: Elements of Reusable Object-Oriented, Addison-Wesley, 1994.

7. Hogia Mobilast home page, http://www.hogia.se/godstrafik/

8. M. Kolp, T. T. Do and S. Faulkner. Introspecting Agent-Oriented Design Patterns, In S. K. Chang (Eds.), Advances in Software Engineering and Knowledge Engineering, vol. III, World Publishing, 2004.

9. Movex home page, http://www.intentia.com/

10. B. Schneier, Secrets \& Lies - Digital Security in a Networked World, John Wiley \& Sons, New York NY, 2000.

11. R. Smith, Authentication: From Password to Public Keys, Addison Wesley Professional, Boston MA, 2002.

12. A.F. Westin, Privacy and Freedom, Atheneum, New York NY, 1968.

13. Zimmermann, R., Butscher, R., Bodendorf, F., An Ontology for Agent-Based Supply Chain Monitoring, Agent Technologies in Logistics, ECAI 2002 Workshop, Lyon, France, 2002. 